Hautarzt 2015 - 66:890-891

DOI 10.1007/s00105-015-3713-y

Online publiziert: 26. Oktober 2015

(c) Springer-Verlag Berlin Heidelberg 2015

CrossMark

\author{
H.-Chr. Schuppe ${ }^{1}$ - G. Haidl ${ }^{2}$ - F. Ochsendorf ${ }^{3}$ \\ ${ }^{1}$ Bereich Andrologie, Klinik und Poliklinik für Urologie, Kinderurologie und Andrologie, Universitätsklinikum \\ Gießen und Marburg GmbH, Justus-Liebig-Universität Gießen, Gießen, Deutschland \\ 2 Universitätsklinikum Bonn-AöR, Klinik und Poliklinik für Dermatologie, \\ Abteilung Andrologie, Bonn, Deutschland \\ ${ }^{3}$ Universitätsklinikum Frankfurt, Klinik für Dermatologie, Venerologie \\ und Allergologie, Frankfurt am Main, Deutschland
}

\title{
Andrologie in der Dermatologie - wozu?
}

de des Interessenspektrums einer Klinik angesiedelt. So wurden von Generation zu Generation - bei mangelnden beruflichen Perspektiven und schwindenden strukturellen Ressourcen - die andrologischen Tätigkeiten stetig zurückgefahren. Dabei sind gerade die Dermatologen prädestiniert, andrologische Probleme bei ihren Patienten zu erkennen, anzusprechen, weiter zu diagnostizieren und ggf. auch zu behandeln. Andererseits erfordert das Aufgabenspektrum der klinischen Andrologie heutzutage eine effiziente interdisziplinäre Vernetzung, was aus unserer Sicht die Attraktivität des Faches erhöht.

\section{) Dermatologen sind prädestiniert, andrologische Probleme zu erkennen}

Vor diesem Hintergrund freut es uns sehr, dass jetzt ein Leitthemenheft „Andrologie" aufgelegt werden kann, mit dem wir die vielfältigen Verwurzelungen andrologischer Fragestellungen im allgemeindermatologischen Alltag aufzeigen wollen. Inhaltlich wird die Andrologie von vielen nur mit der Diagnostik und Therapie männlicher Fertilitätsstörungen assoziiert, jedoch können andrologische Aspekte dem praktisch tätigen Dermatologen auch in anderen Zusammenhängen vielfach begegnen.

Schon bei der klinisch dermatologischen Untersuchung können Befunde wie eine Gynäkomastie oder eine Varikozele auffallen und Anlass für eine spezielle weitere Beratung sein (S. Volc, S. Schanz,
Andrologisch relevante Befunde aus der Sicht des Dermatologen).

Ein sehr aktuelles Thema in der Andrologie ist der altersbedingte Hypogonadismus, der u. a. auch mit dem metabolischen Syndrom auftritt. Forschungsergebnisse der letzten Jahre zeigen, dass auch zwischen metabolischem Syndrom, Hypogonadismus und Psoriasis ein potenzieller Zusammenhang besteht (D. Varwig-Janßen, Der "dicke" und der "alte" Patient in der dermatologischen Praxis: Wann muss man an Hypogonadismus denken?). Hieraus ergeben sich relevante Fragestellungen, mit denen sich auch der nichtandrologisch tätige Dermatologe auseinandersetzen muss, nicht zuletzt im Hinblick auf eine wirksame Primär- und Sekundärprävention.

Dass bei ausgedehnten Dermatosen neben dem Allgemeinbefinden auch die Sexualität beeinträchtigt sein kann, ist belegt. Patienten mit Psoriasis haben häufig Erektionsstörungen und wünschen sich ärttliche Hilfe, die sie aber meist nicht erhalten. Hier hilft es, Berührungsängste abzubauen (F.-M. Köhn et al., Dermatologische Erkrankungen und Sexualität. Wie geht man vor?).

Es sind viele neue medikamentöse Behandlungsverfahren in die Dermatologie eingeführt worden, die potenziell Auswirkungen auf die männliche Fertilität oder Sexualität haben können. Teilweise muss dabei auch auf die Option bzw. Notwendigkeit einer Kryokonservierung von Spermien vor Behandlungsbeginn hingewiesen werden, insbesondere wenn eine Gefährdung des Erbguts zu befürchten ist. Beispielhaft angeführt seien hier $\mathrm{Zy}$ - 
tostatika, Immunsuppressiva und antientzündliche Agenzien wie Methotrexat, zu berücksichtigen sind aber auch einige Biologika (S. Grunewald, U. Paasch, Medikamentöse Therapie dermatologischer Erkrankungen. Wann muss ein Androloge wie beraten?).

Natürlich gibt es auch „Kolibris“ in der Andrologie, mit denen man sich qualifiziert auseinandersetzen muss: So stellt die Problematik der Spermaallergie eine Herausforderung für jede dermatologisch-allergologische Abteilung dar (J.-P. Allam et al., Spermaallergie).

Die korrekte Interpretation eines Spermiogramms sollte jedem Dermatologen geläufig sein. Dies erwartet man z. B. auch bezüglich der Syphilisserologie - obwohl einem beide Fragestellungen nicht unbedingt täglich begegnen ( $T$. Weberschock et al., Spermiogramm bei unerfülltem Kinderwunsch. Was sagt es aus?).

Wir hoffen, dass dieses Leitthemenheft so interessant für Sie zusammengestellt ist, dass Sie (wieder) beginnen, sich intensiver mit der Andrologie zu beschäftigen. Mögen die Beiträge Sie dabei anregen und mit praxisrelevanten Informationen adäquat unterstützen.

Viel Vergnügen bei der Lektüre!<smiles>CCCCCCCCCCC</smiles>

G. Haidl

\section{g. Fell Ochunaf}

F. Ochsendorf<smiles></smiles>

H.-Chr. Schuppe

\section{Korrespondenzadresse}

\section{Prof. Dr. G. Haidl}

Universitätsklinikum Bonn-AöR

Klinik und Poliklinik für Dermatologie,

Abteilung Andrologie

Sigmund-Freud-Str. 25

53127 Bonn

gerhard.haidl@ukb.uni-bonn.de

\section{Prof. Dr. F. Ochsendorf}

Universitätsklinikum Frankfurt

Klinik für Dermatologie, Venerologie und Aller-

gologie

Theodor-Stern-Kai 7

60590 Frankfurt am Main

Ochsendorf@em.uni-frankfurt.de

\section{Prof. Dr. H.-Chr. Schuppe}

Bereich Andrologie, Klinik und Poliklinik für Urologie, Kinderurologie und Andrologie Universitätsklinikum Gießen und Marburg GmbH, Justus-Liebig-Universität Gießen Gaffkystr. 14, 35392 Gießen Hans-Christian.Schuppe@ derma.med.uni-giessen.de

Interessenkonflikt. H.-Chr. Schuppe, G. Haidl und F. Ochsendorf geben an, dass kein Interessenkonflikt besteht.

\section{Literatur}

1. Köhn FM (2014) Weißbuch Andrologie 2014 des Arbeitskreises Andrologie der DDG (Bezug über F.M. Köhn, info@andrologicum.com)

2. Krause W (2012) Andrologie in der Dermatologie: Entstehung und Entwicklung. Derm 18:99-106 